\title{
Data Storage, Processing, and Visualization for the Australia Telescope Compact Array
}

\author{
Tara Murphy ${ }^{\mathrm{A}, \mathrm{C}, \mathrm{D}}$, Peter Lamb ${ }^{\mathrm{B}}$, Christopher Owen ${ }^{\mathrm{C}}$, and Malte Marquarding ${ }^{\mathrm{C}}$ \\ A School of Physics, University of Sydney, Sydney NSW 2006, Australia \\ ${ }^{\text {B }}$ CSIRO ICT Centre, Canberra ACT 2601, Australia \\ C Australia Telescope National Facility, Epping NSW 1710, Australia \\ D Corresponding author. Email: tara@physics.usyd.edu.au \\ Received 2005 October 1, accepted 2006 January 14
}

\begin{abstract}
We present three Virtual Observatory tools developed at the Australia Telescope National Facility (ATNF) for the storage, processing and visualization of Australia Telescope Compact Array (ATCA) data. These are the Australia Telescope Online Archive, a prototype data-reduction pipeline, and the Remote Visualization System. These tools were developed in the context of the Virtual Observatory and were intended to be both useful for astronomers and technology demonstrators. We discuss the design and implementation of these tools, as well as issues that should be considered when developing similar systems for future telescopes.
\end{abstract}

Keywords: astronomical data bases: miscellaneous — methods: data analysis

\section{Introduction}

The data explosion in astronomy promises exciting new scientific developments, but brings with it many technical challenges in collecting, storing, transporting, processing, and visualizing data. Virtual Observatories (VOs) have developed to meet some of these technical challenges. Falling under the broad area of e-science (which incorporates other scientific domains facing similar challenges, such as genetics and particle physics) the aim of Virtual Observatory research is to provide the tools necessary for dealing with this data.

The Australian Virtual Observatory (Aus-VO) ${ }^{1}$ was started in 2003 with the aim of both contributing to the international VO effort, and developing tools of use to Australian astronomers. Australia has many areas of expertise (for example radio astronomy) and it makes sense to focus our efforts on providing modern tools for working in these areas. In this context the ATNF decided to develop a range of tools for storing, processing, and visualizing data from the Australia Telescope Compact Array (ATCA). The aim was that these tools would be useful to astronomers now, and at the same time let us explore the technology that would be necessary for developing software for future telescopes such as the Square Kilometre Array (SKA).

This paper is based on a talk given at the ASA Annual Meeting in Sydney in 2005 July. After giving some background about the ATCA and the Virtual Observatory, we discuss three tools developed at the ATNF over the last few years - the Australia Telescope Online Archive which contains all of the data collected so far by the ATCA, a

${ }^{1}$ www.aus-vo.org prototype data-reduction pipeline for ATCA data, and finally the Remote Visualization System for viewing large datasets.

\section{The ATCA}

The ATCA is an east-west Earth-rotation synthesis interferometer, with six 22-m antennas on a 6-km baseline. It has been in operation at Narrabri since 1990. The telescope can observe at six bands with wavelengths $20 \mathrm{~cm}$, $12 \mathrm{~cm}, 6 \mathrm{~cm}, 3 \mathrm{~cm}, 1 \mathrm{~cm}$, and $3 \mathrm{~mm}$. Each antenna observes two frequencies simultaneously. There are six bandwidths available on Frequency 1 (128, 64, 32, 16, 8, and $4 \mathrm{MHz})$ and two bandwidths on Frequency 2 (128 and $64 \mathrm{MHz}$ ). The telescope produces about $0.5 \mathrm{~GB}$ of raw data per day, and this is likely to increase significantly with future telescope upgrades.

In the rest of this section we outline the existing systems for archiving, processing and visualizing ATCA data.

\subsection{Data Archiving}

Since the commencement of operation of the ATCA in 1990 June, a complete record of all data observed from the telescope has been maintained offline at the telescope site, mostly recorded on CD. In conjunction with this, the ATNF maintained a record of the project proposals for observations on the telescope - the Projects Database — and a short form of the observation parameters for each day's observing — the Positions Database. After a proprietary period of 18 months, in which the observing team has sole access to the data obtained in an observation, the data is made publicly available. Astronomers can search for 
observations on the ATNF webpage, submit the details of the observation data required through e-mail, and have a $\mathrm{CD}$ containing the data prepared for them at nominal cost.

\subsection{Data Processing}

ATCA data processing (reduction) is generally performed with one of the standard radio data reduction packages, most commonly MIRIAD (Sault et al. 1995) but also AIPS $^{2}$ and AIPS++ ${ }^{3}$. After loading, editing, and calibrating the data, the resulting product is an intensity map referred to as the dirty image. At this stage a deconvolution algorithm, usually a variant of CLEAN (Högbom 1974), is required to produce the final image.

At each stage in the process there are a range of parameters that can be set to control the type of processing performed. Both general parameters (such as calibration strategy, CLEAN method, and type of data editing), as well as fine-grained parameters (such as calibration solution interval, number of CLEAN iterations, and median filter size) need to be modified to obtain the best results. Hence data processing is typically a highly interactive process.

There is an existing system operating at the telescope CAONIS designed for on-the-fly imaging of ATCA data. However the design of this system makes it difficult to port to current Linux systems.

\subsection{Data Visualization}

The final images from the ATCA are usually visualized using tools such as MIRIAD or KVIS (Gooch 1996). These are well established tools that cover many of the visualization requirements of ATCA observers. As mentioned in the previous section, the CAONIS system, which runs at Narrabri, also allows basic visualization of images.

\section{The Virtual Observatory}

The umbrella organization for Virtual Observatory work is the International Virtual Observatory Alliance (IVOA). The IVOA was formed in 2002 June with a mission to

'... facilitate the international coordination and collaboration necessary for the development and deployment of the tools, systems, and organizational structures necessary to enable the international utilization of astronomical archives as an integrated and interoperating virtual observatory.'

The IVOA is a collaboration between over 15 member countries including Australia. The focus so far has been developing the standards required for interoperability between software developed and data produced in all areas of astronomy. Another significant aim is to develop the infrastructure required (networks and organizations) for the large scale storage and distribution of astronomical data.

The IVOA working groups address a range of issues such as grid and web services, data modelling, and

\footnotetext{
${ }^{2}$ Astronomical Image Processing System: www.cv.nrao.edu/aips

${ }^{3}$ Astronomical Image Processing System: aips2.nrao.edu
}

standards for the data access. There are also four interest groups

- Applications IG

- Astronomy Grid IG

- Data Curation IG

- Theory IG

that focus on the requirements of particular application domains.

The aim of the Australian Virtual Observatory (Aus-VO) is to provide distributed, uniform interfaces to the data archives of Australia's major observatories and the archives of simulation data. Aus-VO is a collaboration between many Australian institutions, including the Universities of Melbourne, Sydney, New South Wales, and Queensland, Monash University, Swinburne University of Technology, the Australian National University, Mount Stromlo Observatory, the Victorian Partnership for Advanced Computing, the ATNF, and the Anglo-Australian Observatory.

There are a range of VO projects underway in Australia, including the development of data archives and software for HIPASS (Meyer et al. 2004), RAVE (Siebert 2004), 2QZ (Croom et al. 2004), and SUMSS (Bock et al. 1999). The initial focus of most of these projects has been to make data from Australian projects widely available within the international community, in a VO-compliant format. In addi-tion there are several projects investigating novel methods for astronomical data mining and data analysis, for example Rohde et al. (2005) applied machine learning techniques to catalogue crossmatching. The University of Melbourne group has also been setting up infrastructure such as a registry for Australian web services and data archives.

\section{The Australia Telescope Online Archive}

In 2003 June, a joint project between the ATNF and the CSIRO ICT Centre was commenced to make the ATNF archive data available online as the Australia Telescope Online Archive (ATOA). This was planned as a new data resource for astronomers, as well as the foundation for the development of online data processing systems to make the raw data more accessible to non-expert users (see Section 5). The construction of the ATOA required the copying of the offline archive (at the time, about 2700 CDs totalling approximately $1.7 \mathrm{~TB}$ ) from the telescope site to Canberra where the online archive was to be developed, creating a meta-database describing the data, and making a web frontend to search and download the data.

Table 1. ATOA statistics

\begin{tabular}{lr}
\hline Projects & 2261 \\
Files & 57147 \\
Sources & 128111 \\
Metadata size & $\sim 4 \mathrm{~GB}$ \\
RPFITS data size & $\sim 2 \mathrm{~TB}$ \\
Growth rate & $\sim 0.5 \mathrm{~GB} \mathrm{~d}^{-1}$ \\
\hline
\end{tabular}


The database consists of two parts. The first is the raw data from the telescope (RPFITS files) which is stored as normal files on the host system. In addition there is a relational database which stores all the metadata (discussed in Section 4.1). The 'vital statistics' of the ATOA are shown in Table 1. The current rate of growth of the archive is circa $0.5 \mathrm{~GB}$ per day. However this is likely to increase significantly in the future as new instruments come online. To maintain an growing archive, rather than a static one, it is necessary to ensure the RPFITS files are stored in a readily accessible way (currently on a RAID system) that is easily distributed over a number of drives. Also, that the database itself is easy to update in a robust manner. The ATOA was made publicly available in 2004 December ${ }^{4}$.

\subsection{Metadata}

Metadata is simply data which describes other data, for example the project code or the name of the primary calibrator. The meta-database for the ATOA consists of three main parts; the contents of the original ATNF online Projects Database, metadata describing the observation that is extracted directly from the raw data files produced by the telescope's software, and metadata inferred from all of the available data sources to assist in the automation of reducing the telescope's raw data to images. The types of metadata used in the ATOA are summarized in Table 2.

Most of the metadata available in the ATNF Positions Database is also available from the metadata in the raw data files and is finer-grained, since the Positions data is a daily summary whereas the file metadata is available for each telescope pointing. The inferred metadata in the ATOA is 'value added' information that is automatically determined from the existing metadata, for example the calibration role of each source (primary calibrator, secondary calibrator, target, etc). This is discussed further in Section 5.2.

Table 2. Metadata in the ATOA

\begin{tabular}{|c|c|}
\hline Metadata source & Examples \\
\hline Projects Database & $\begin{array}{l}\text { proposal } \\
\text { observer name } \\
\text { country } \\
\text { institution }\end{array}$ \\
\hline Positions Database ${ }^{\mathrm{A}}$ & $\begin{array}{l}\text { source names and positions } \\
\text { observing band } \\
\text { receivers }\end{array}$ \\
\hline RPFITS files & $\begin{array}{l}\text { scans and polarizations } \\
\text { array configuration }\end{array}$ \\
\hline Inferred & $\begin{array}{l}\text { calibrator names and roles } \\
\text { calibrator-target matches }\end{array}$ \\
\hline
\end{tabular}

\footnotetext{
A The Positions Database is included in our data model, and some of the metadata is used to reconstruct the observation metadata. However, it is not actually loaded into the ATOA.
}

\footnotetext{
${ }^{4}$ atoa.atnf.csiro.au
}

\subsection{A Data Model for the ATCA}

A data model is a comprehensive scheme describing how data is to be represented, for manipulation by humans or computer programs. Data models are critical for planning how data will be organized within a database as they describe all the relationships between the different entities.

A section of our data model for the ATCA is shown in Figure 1. We now briefly explain the UML (Unified Modelling Language) notation used in the data model. Each box contains an entity (e.g. Scan) that has been identified in the metadata. Each entity has attributes (e.g. restFreq), each of which are of a specified data type (e.g. float). Associated entities are connected to each other with lines, which also specify the cardinality of the relationship. For example

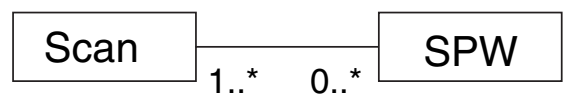

should be read as 'A scan has 0 or more spectral windows. A spectral windows has 1 or more scans.'

The development of a data model that covers the whole of astronomy is an ongoing project within the international VO community. We have contributed this data model to the IVOA Data Model WG as an example of a data model for radio astronomy. For more information on this topic, see the IVOA Data Modelling website ${ }^{5}$.

The ATOA archive database structure is created directly from the definitions in the ATOA data model. Parts of the data model contain information for specific database implementations so that all of the implementation-specific parts of the database creation are handled in this process. The data model in Figure 1 corresponds to the part of the data model that describes the metadata contained directly in the archive RPFITS files. The data model for the inferred data is available from the ATOA web pages ${ }^{6}$.

\subsection{Implementation}

The ATOA web interface was implemented as a Java (ver. 1.4.2) ${ }^{7}$ application and is hosted using the Apache TOMCAT (ver. 5.0.28) ${ }^{8}$ web container. Relational database services are provided by an ORACLE 9I instance running on the same machine. A web-based interface was chosen so as to maximize interoperability and provide easy access to users. For example, RPFITS files may simply be downloaded by constructing a suitable URL for the ATOA file server. This allows files on the server to be downloaded by a Web browser, by command-line programs that allow users to fetch the data referred to by a URL, or by application programs using libraries that allow a URL to be opened in a similar way to a file on a local file system.

The user interface centres around two main web pages: the query page which allows users to specify criteria for

\footnotetext{
5 www.ivoa.net/twiki/bin/view/IVOA/IvoaDataModel

${ }^{6}$ www.atnf.csiro.au/computing/web/atoa/implementation.html

7 java.sun.com

8 tomcat.apache.org
} 


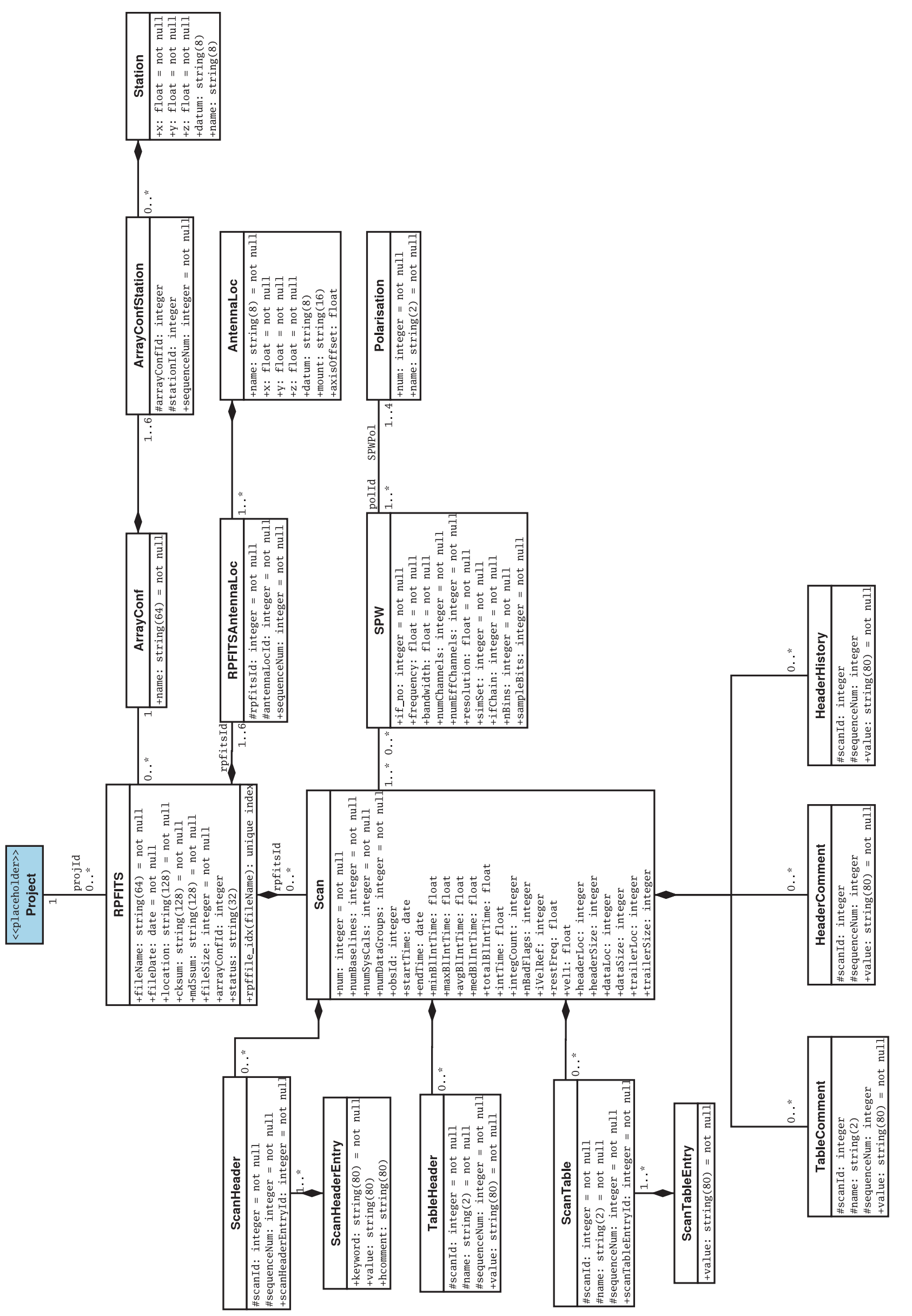


selecting RPFITS files from the archive, and a results page which provides the means for users to inspect the metadata of matching files and download particular files if desired. The results page initially presents the user with a broad, global view of the query results in tabular form listing details such as file name, file size, principal investigator, and array configuration. The user may also interactively ‘drill-down' for a more detailed view of any file in the list. RPFITS files can be downloaded individually or in batches.

As mentioned in Section 2.1 ATCA data has a proprietary period of 18 months, in which it is only accessible to members of the project team. Authorized access is supported for data within the proprietary access period. If a user wishes to access proprietary data they must first go through a manually verified authentication process after which a password is issued to the Principal Investigator for that project. In the future we plan to replace this authentication and authorization method with a streamlined system that links the new ATNF proposal system, $\mathrm{OPAL}^{9}$, and its authentication database to the ATOA. Users will then be given access to proprietary data by using their OPAL credentials based on the projects they are associated with in the OPAL system.

The ATOA web server and database are hosted on a Dell PowerEdge 750 running Debian Linux 3.0. The host has a $2.8 \mathrm{GHz}$ Pentium 4 processor, $2 \mathrm{~GB}$ of RAM and is attached to a 3 TB Apple Xserve RAID for archive storage.

\section{A Data Processing Pipeline Framework}

The data products in radio astronomy are often less accessible to the non-expert than those in other domains such as optical astronomy. It requires a reasonably high level of domain expertise to process the raw data and produce an image. Obviously for carrying out detailed scientific analysis it would be necessary to develop this expertise or collaborate with a radio astronomer. However in an era of multiwavelength astronomy, astronomers expect to download and compare data from a variety of telescopes taken at a variety of wavelengths.

With this in mind we have developed an automatic pipeline for people who want to quickly inspect the data in the ATOA, to see if it was suitable for further processing. One of the aims of this project was to test the viability of 'driving' the pipeline using the metadata discussed in Section 4.1. In other words the pipeline should make decisions about what kind of processing to do - both on a general (e.g. continuum/spectral line) and specific level (e.g. number of CLEAN iterations).

In this section we discuss the development of extra metadata required to driving the pipeline, in particular the calibration process. We then outline our prototype pipeline which can process single-pointing continuum data from the ATOA and is available for testing ${ }^{10}$.

\footnotetext{
9 opal.atnf.csiro.au/

${ }^{10}$ atoa.atnf.csiro.au/test
}

\subsection{Metadata for Automatic Processing}

The metadata in the Project and Position Databases, while providing information about which astronomical sources have been recorded in an observing session, does not (in general) provide any information about the role that the observer intended the source to play in the observation (e.g. primary calibrator, target source). This would be relatively easy to record in a new system, but as we are dealing with existing data we had to infer the roles of sources.

Another problem for automatic processing is the grouping of data into valid 'observations'. An expert would typically choose an appropriate subset of files from the archive to image. However, a non-radio astronomer may choose an subset that contains files that should be imaged separately, or files that contain data that should be ignored entirely. Although it is impossible to deal with all cases, our aim was to have the pipeline group together the selected data in such a way that an image could be made in at least $80 \%$ of cases. A wide range of observation types can be recognized and characterized using the meta-database but are not yet processed by the prototype pipeline (e.g. millimetre and spectral line observations).

In the following section we discuss how we assign the source roles within an observation, and the algorithm we used to match target sources with the appropriate calibrators.

\subsection{Determining Source Roles}

Matching target sources with their calibrators would be a straightforward task for an astronomer but is a challenge for an automatic system. In a typical (simple) observing session the primary calibrator is recorded for a short period at the start or end of the observing session and alternating pointings are made to the secondary (source of interest, target). However there is a great variety of different ways that the observer can choose to structure their observations. If an observation contains more than one target the targets may share, or have distinct, secondary calibrators depending on their separation in the sky. There may be several secondary calibrators for each target, and the same source may be used for primary and secondary calibration. In addition, some observers use secondary calibrators that are not in the list of recommended calibrators, and that list has itself changed over time.

In order to classify the sources in an observing session the following metadata is used:

- The locations and names of sources extracted from the raw telescope data

- The times and durations of the source pointings

- The names and locations of the four primary calibrators commonly used at the ATCA

- A recent ATCA catalogue of recommended secondary calibrators

- Names of sources extracted from project titles

- A pre-assembled list of possible calibrator sources

Once the source roles have been determined, the proximity in the sky and the proximity in observation 
time of the targets and their secondary calibrators are used to match targets with their respective calibrator(s). For each target pointing, a weight is calculated for each secondary calibration pointing made within two hours of observation of the target pointing:

$$
\begin{aligned}
& w_{t, s}=\Sigma_{P} \Sigma_{S} \exp \left[-\left(3 a / a_{\max }\right)^{2} / 2\right] \exp \left[-\left(3 \Delta t / \Delta t_{\max }\right)^{2} / 2\right] \\
& \Delta t_{t, s}<\Delta t_{\max }
\end{aligned}
$$

where $S$ is the set of candidate secondary calibrators, $a$ is the angular separation between the target and the secondary calibrator, $a_{\max }$ is the maximum desirable separation between the target and the secondary calibrator (and is a function of the observing frequency band), $\Delta t$ is the separation of the time midpoints of the target and calibrator pointings, and $\Delta t_{\max }$ is the maximum desirable time separation (two hours). The summation is over all pointings at a target $\left(\Sigma_{P}\right)$ and all secondary calibrators within two hours of a target pointing $\left(\Sigma_{S}\right)$. The $w_{t, s}$ weights are used to select suitable secondary calibrators for the respective targets from the calibrators whose $w_{t, s}$ weights dominate for a particular target.

This procedure constructs the metadata required for continuum imaging at centimetre wavelengths. The algorithm works well in general but there are some problematic cases, for example where the target is a source from the secondary calibrator catalogue.

\subsection{Implementation}

The underlying processing of the ATCA data is carried out using the Glish scripting language in AIPS++. The ATOA imaging Web Services interface was constructed using the Apache AXIS tools (ver. 1.1) ${ }^{11}$, and interfaces to the processing scripts through a Perl (ver. 5.4.8) ${ }^{12}$ script that deals with the control of the execution of the Glish scripts.

The pipeline client is written using Python (ver. 2.3 $)^{13}$, and the SOAPPY web services tools (ver. 0.11.3 $)^{14}$. There were some minor, but difficult to find, problems in interoperation between the SOAPPY tools and Apache AXIS; the data structures used in the web services calls are possibly more complicated than had been previously used between the two web services implementations. Documentation in both was not as informative or complete as it might have been.

The pipeline web services can be configured to run directly on the server host, or be directed to run on other machines through a batch queuing system, since some stages in the PIPELINE can run for several CPU minutes. We used the OPENPBS batch queuing system (ver. 2.3) ${ }^{15}$ for queue management but unfortunately it has no mechanism for reporting job completion to another program. After processing for a web service completes, the batch job doing the processing sends a completion

\footnotetext{
11 ws.apache.org/axis

12 www.perl.com

13 python.org

14 pywebsves.sourceforge.net

15 www.openpbs.org
}

message to the program invoked by the web service that controls the execution of the processing for the service. However, at this point, the batch processing system has not yet transferred the job's output data back to the pipeline server. The control program then polls the PBS batch queue at five-second intervals to ensure that the batch job has completed.

The raw data from the ATOA, all the intermediate files from the data processing, the log files, and the resulting images are stored temporarily on the pipeline server. The first web service call made by a pipeline client reserves a private location for storage and requests a lifetime for the storage. The pipeline server has a configurable maximum lifetime, and the stored data will be deleted after this time expires. Only clients who have the name of the storage area (a randomly generated string) can access it. There is no quota on the storage use of any individual temporary storage area. However, a quota may be imposed on the total amount of storage available to all active storage areas.

The ATOA and pipeline web services return a URL for the generated images to the end-user's system. This allows the URL to be passed on to the Remote Visualization System (see Section 6) image viewing system so that the image can be viewed online while it is still resident on the pipeline server. Figure 2 show the overall system architecture, in particular how the ATOA, pipeline, and RVS interact.

\section{The Remote Visualization System}

The Remote Visualization System (RVS) was designed to enable visualization of and interaction with large astronomical images in the context of the VO. As opposed to other VO image displays, such as CDS Aladin (Fernique et al. 2004), RVS does not require the user to download the data to the client machine. Furthermore it

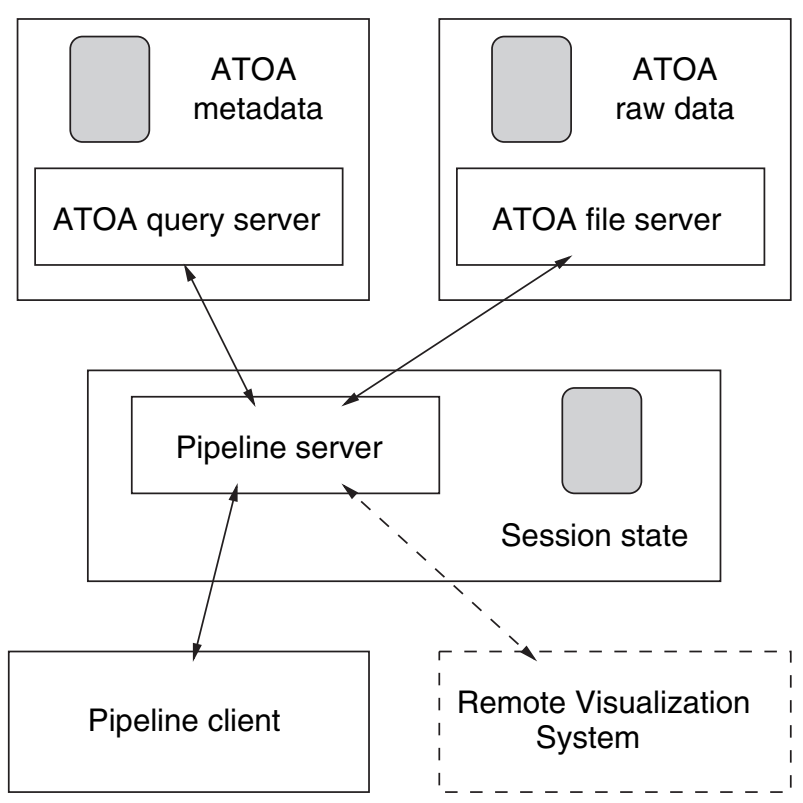

Figure 2. System architecture. This schematic shows the relationship between the three tools we have developed. 


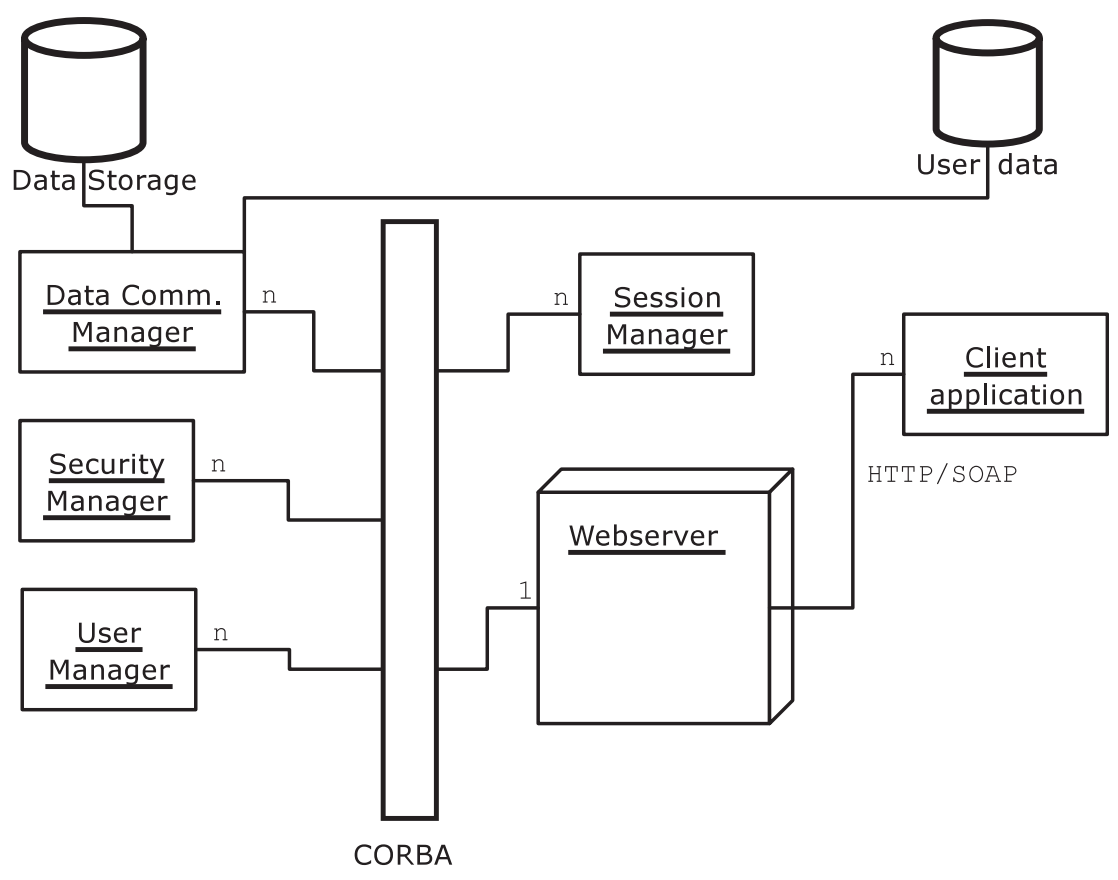

Figure 3. RVS system architecture.

provides rendering of image cubes, such as spectral-line cubes created from ATCA data. The RVS server accepts FITS images - which can be compressed - through local URLs and remote HTTP or FTP access. The data should be co-located with or at least be available to the server on high bandwidth connection, while it places no such requirements on the client. Only minimal data transfer to the client is necessary and this is independent of the size of the source data set. The server-side architecture is distributed to enable workload sharing and extensibility. RVS makes use of several software components: CORBA to make it distributed, AIPS++ as the image rendering component, and Java for the web services and client. The architecture of the RVS system is shown in Figure 3.

RVS is exposed through a web service interface using the standard Web Service Description Language (WSDL ver. 1.1) ${ }^{16}$. This can easily be integrated into custom applications. Several client applications make use of the web service interface: the RVSVIEWER — a traditional image viewer and thumbnail service — providing preview images and a session viewer. The session viewer connects to an existing RVSVIEWER by means of a key. Multiple instances can be run at the same time, making it a possible to use it as a conferencing tool where people can observe and interact with the data. The ATOA pipeline re-uses the existing RVSVIEWER client by passing it the file location of the output image.

RVS is not specific to the ATOA or prototype pipeline and there are plans to use it for all ATCA archives. It has been successfully tested on images and data cubes from various surveys and has good performance on large datasets. For example a $1.5 \mathrm{~Gb}$ data cube from the Galactic All-Sky

${ }^{16}$ www.w3.org/TR/wsdl
Survey (GASS; McClure-Griffiths et al. 2005) takes about one minute to load. Compare this with downloading the full cube from say the USA to Australia which could take about one to two hours. For more information and direct access to RVS, see our website ${ }^{17}$.

\section{Discussion}

The ATOA has been public since 2004 December. We hope that it will encourage the reuse of ATCA data for projects other than those it was originally intended for. The framework used for the ATOA could easily be extended to include data from other telescopes and can be updated as additional metadata is required.

The most significant improvement of the ATOA over existing online archives (such as NRAO ${ }^{18}$ and $\mathrm{MAST}^{19}$ ) is the data delivery mechanism. Most existing archives do not support on demand delivery of data over the web, instead requiring the user to submit a form requesting files that then have to be transferred to a publicly accessible FTP site or to other media (such as CDs) for physical delivery. In the ATOA, the batch downloading of multiple files is handled by a streaming TAR or ZIP archiving algorithm that performs dynamic archiving as files are streamed over the web, requiring no additional disk space on the server for these operations.

In developing the ATCA data model and considering the type of metadata required for automatic processing we identified several new metadata types that would be useful to store in the RPFITs files. As a result the following

\footnotetext{
17 atnf.csiro.au/vo/rvs/

18 archive.nrao.edu/archive/e2earchive.jsp

19 archive.stsci.edu/
} 
fields have been added to the RPFITS files and will be available in all future ATCA data:

- Four calibrator codes

C (standard phase calibrator)

F (primary flux calibrator)

B (bandpass calibrator)

$\mathrm{P}$ (pointing calibrator)

- Pointing offsets

- Weather data: added rain gauge and phase RMS and difference

- Attenuator settings at start of scan

- Subreflector position

- Correlator configuration

- Scan type

- Coordinate type

- Line mode

- CACAL counter

These will help both automatic processing systems and astronomers assess the data quality in the observations they are interested in. A full e-logbook system will be used in the future as currently the logs are all stored on paper at the telescope and hence are not easily accessible to ATOA users.

We have developed a prototype pipeline for processing of raw data for single-pointing continuum images. This is attached to the ATOA to provide an improved service for users of the ATOA. At this stage the image quality is suitable for previewing the data in archive to see if it is of interest. Further manual processing would then be required to obtain images of scientific quality.

A significant challenge in developing the ATOA and the prototype pipeline were integrating pre-existing software with modern software tools. For example, the Glish scripting language has no web service libraries and so an extra layer had to be developed between the data processing level and the web services. If re-implementing from scratch, a language such as Python would be a better alternative for developing the pipeline.

In developing these tools we have started to explore the techniques necessary for astronomical software development in the VO era. This is essential for future telescopes and surveys that Australia will produce. Making access to existing Australian data as easy as possible will maximize its use in the international community.

\section{Acknowledgments}

The authors would like to acknowledge the software development done on the RVS project, primarily by Anil Chandra and also by Praveena Tokachichu. The ATNF side of the prototype pipeline and ATOA development was managed by Neil Killeen and Jessica Chapman. Vince McIntyre contributed extensively to all three projects, in particular in setting up the hardware required.

A number of ATNF staff put in significant effort to get the ATOA set up, in particular Robin Wark, Bob Sault, and Mark Wieringa. Warwick Wilson and Mark Wieringa implemented the changes to add extra metadata to the RPFITS files.

From the ICT Centre, Robert Power made the initial data model designs, the ATOA data loader software and ATOA query front end. Geoff Squire and Bella Robinson made significant contributions to the prototype pipeline.

\section{References}

Bock, D. C.-J., Large, M. I., \& Sadler, E. M. 1999, AJ, 117, 1578 Croom, S. M., et al. 2004, MNRAS, 349, 1397

Fernique, P., et al. 2004, in Toward an International Virtual Observatory (Eds Quinn, P. J., \& Górsky, K. M.) p. 271 (Heidelberg: Springer)

Gooch, R. 1996, in ASP Conf. Ser. 101: Astronomical Data Analysis Software and Systems V (Eds Jacoby, G. H, \& Barnes, J.) p. 80 (San Francisco: ASP)

Högbom, J. A. 1974, A\&AS, 15, 417

Meyer, M. J., et al. 2004, MNRAS, 350, 1195

McClure-Griffiths, N. M., et al. 2006, ApJ, in press (astro$\mathrm{ph} / 0510304)$

Rohde, D. J., et al. 2005, MNRAS, 360, 69

Sault, R. J., Teuben, P. J., \& Wright, M. C. H. 1995, in ASP Conf. Ser. 77: Astronomical Data Analysis Software and Systems IV (Eds Shaw, R. A, Payne, H. E., \& Hayes, J. J. E.) p. 433 (San Francisco: ASP)

Siebert, A. 2004, in SF2A-2004: Semaine de l'Astrophysique Francaise (Eds Combes, F., Barret, D., Contini, T., Meynadier, F., \& Pagani, L.) p. 567 (Les Ulis: EdP Sciences) 